\title{
Models and modeling with the Louisville Experiment Simulation System (LESS)
}

\author{
JOHN B. THURMOND and ARTHUR O. CROMER \\ University of Louisville, Louisville, Kentucky 40208
}

\begin{abstract}
A system is described which permits the average instructor, without programming knowledge, to prepare a simulation model that can be called up by students as a simulated experiment.
\end{abstract}

Several years ago, we replaced the required undergraduate course in experimental psychology, which emphasized only the theory of research, with a course that emphasizes practice as well. This new problem-solving approach uses computer simulations as an aid to learning the concepts of good research strategy. In order to implement the approach, we created three computer models of "relevant" problems in the areas of human memory, selective attention, and obesity. The student is introduced to the problem area by reading appropriate background material. He is then presented with a list of potentially important variables in the problem area and told to choose how he would collect data relevant to the problem area if he could make choices among the variables.

The student selects the variables he wishes to investigate and the specific levels of these variables; the computer model calculates the results, presenting the data as if the specified experiment had just been run. The data produced by the computer contains random error, as would be the case if a "real" experiment had been conducted; thus, the results of an experiment run on the computer vary, even if the same experiment is replicated. Along with this output, the programs give an "experimental cost" based on the parameter values chosen. The student then may ask for summary statistics and the error of this run as compared to the preceding run, to aid in analysis. By combining the simulations with live experiments, where the emphasis is on the techniques necessary for running the experiments and collecting the data, a complete balanced coverage is provided for the entire range of research training. This technique has been used successfully in about 30 sections (20 students each) of the experimental psychology courses offered at the University of Louisville since the fall of 1971.

Supplementing course content, demonstrations, and laboratory experiments with data-generating models is a new use of computers, and it improves instruction in at least four ways: (1) Computer models simulate different experimental situations or other situations without the need of expensive equipment and without consumption of valuable time in the class or laboratory. (2) They enhance the student's understanding of the content of the course by permitting him to conduct many experiments in different areas covered by the subject matter of the course. (3) They teach the student to use good scientific research strategy by putting him in a situation where me must formulate hypotheses in order to obtain meaningful results and where he must examine results carefully in order to draw meaningful conclusions. (4) Finally, they increase motivation by involving the student in an active learning process in which he interacts with the computer model in order to understand the phenomena under study.

The main purpose of LESS is to permit instructors in any discipline to use data-generating models in their classes in order to greatly expand the student's learning experience. The data generated by a model used in class are not intended to replace the traditional methods of teaching, e.g., class lectures, demonstrations, and experiments. Rather, the computer-generated data allows instructors to provide their students with an experience with the phenomena dealt with in the course that would not otherwise be possible. Typically, there is time in a course for only a few experiments or demonstrations of some of the more important effects of the phenomena covered.

The student acquires an isolated experience in the usual laboratory experiment, an experience with the effects of variables operating under one rigid set of conditions. Narrow though it may be, this first-hand experience is of crucial importance for the student's appreciation of some of the more important aspects of the course's content. The advantage of using a data-generating computer model to accompany laboratory exercises and demonstrations is that it expands the student's experience to include the entire range of conditions in the cause and effect relationships associated with the phenomena under study. Since the variables that are specified for conditions in the data-generating model are the same as those covered by the content of the course, the student can, if he desires, replicate his laboratory experience by specifying the conditions of his experiment to the computer and then go on to investigate many other conditions as well. Use of the computer to generate the data eliminates the time-consuming aspects of the laboratory procedures. 
The conditions under which the variables are to have effects are specified to the computer, and the data are generated all in a matter of minutes.

All one needs to build such a computer model is a description of the cause and effect relationships relating changes in the variables under study to the data typically obtained. This does not mean that one must understand the process involved by which the variables produce the effects; one only needs to have some idea of what the data should look like, granted that certain conditions are specified. One need not know computer programming in order to build a data-generating model and to use it in a course. The modular system of LESS permits a nonprogrammer to build up and revise his own set of models for his course. Since new models can easily be added or old ones removed, the instructor can start with one simple model to accompany a laboratory experiment, replace it later with a better one, exchange it for a new one, or add models to accompany other content areas of the course. Detailed instructions for nonprogrammers on how to design a model and add it to LESS are given in the Author's Guide to LESS; instructions are also provided for the other mode of building models with the system-that in which the author is a programmer who desires to write his own program to generate data. In order to facilitate these explanations, there is also available a Manual for Users of $L E S S$ which accompanies the author's guide.

LESS is a set of four core programs (LESS, LESSIN, LESPUT, and LESTES) and a data file (LESDAT). The data file, LESDAT, is initialized by running LESSIN. Then, after carefully planning a data-generating model, a session of $10-30$ min using LESPUT is required to add the model to the system. It may then be tested using LESTES, or deleted by LESSIN in order to make major changes by reentering the model. The user (student) is only aware of one program, LESS, which he calls up and runs. This main program calls up whichever model the user selects by chaining to the appropriate model program.

\section{GENERAL MODEL BUILDER}

An important feature of the system is the ability for a nonprogrammer to add a model to the system using the general model builder (GMB). In a second mode, the author is expected to write his own program to generate data. In both modes, the LESPUT program asks a series of identical questions about general characteristics of the model, such as how many variables there will be, what values will be "legal" or recognized for each variable, etc. When the program reaches the point where it begins asking for values that will be used as effects or costs in the data generator, in Mode 1 the questions are very simple. The author is asked how many costs and how many effects values there will be and then is asked to give them. From there on, the two modes are nearly identical. In the second, or general model builder mode, the questions are more elaborate.

The decision as to whether or not to write your own model depends upon more than just whether you are a competent programmer. If you can express your model as the sum of a weighted combination of terms (the GMB will accept up to a 7-way interaction) plus a constant, then it is probably easier to use the general model builder. However, if your model requires a series of logical branches of varying complexity and depth, it is probably more efficient, both of execution time and computer core requirements, to write your own model. An advantage of writing your own model is that you can do almost anything you want to do before returning to the main program. The main program serves the function of accepting variable specifications from the user, checking them for legality and proper form, and passing the "sanitized" specifications to your model. It also can output simple statistics (mean, variance, and correlations) before cycling again to accept more specifications or start another model.

The system handles a number of different types of variables. Each variable can be controlled or randomized by the author of the model to have from no effect to a great effect. In addition, a cost function may be used to approximate the cost of running particular variables or combinations of variables.

Parameter variables are general variables, such as number of cases, sample size, number of time periods, etc., which determine how many values of a dependent variable will be generated in one run.

Discrete variables must take on whole number values only, where the number may just be a label of a category, such as $1=$ male, $2=$ female, or may have more meaning, such as $1=$ below $\$ 5,000$, $2=\$ 5,000-\$ 10,000,3=\$ 10,000-\$ 20,000$, etc.

Continuous variables may be used in an author-written model program directly or, in the GMB mode, may be broken up into discrete sections before use.

$X$ variables are unknown variables which may represent one or more specific variables important to the model which the students are to discover, or may be any variable(s) the author did not include in the original model.

Secondary variables are the specific variables mentioned above which are important to the model but were not originally revealed to the student until he had examined the relationships among the initial set of variables.

Dependent variables represent the results of the measurements or observations on the simulated experiment.

The system, as distributed, already contains six models. Four of these models were developed at the University of Louisville (Thurmond \& Cromer, 1972) and pertain to the areas of fear and sex, human obesity, 
selective attention, and the tip-of-the-tongue phenomenon. The two others were developed at the University of Michigan (Main \& Head, 1971) and pertain to schizophrenia and imprinting. The Student Guide to LESS (published by Xerox College Publishing) is designed specifically for using these six models in a course and is made available by the Campus Store at the University of Louisville. An Instructor's Guide to LESS accompanies the student guide and is duplicated, as needed, with in the department. The system is available in BASIC and FORTRAN and a Programmer's Guide to LESS is available to accompany it.

Of the systems presently available, LESS is intermediate both in terms of complexity and in terms of the degree of sophistication in the models that one can build with the general model builder. The other available systems are either less complex than LESS (Shure, 1975) or more complex and sophisticated (Stout, 1975).

\section{EXAMPLE USING GMB}

The situation of success in class depends upon several variables. For simplicity, we will consider only ABILITY and EFFORT. We will allow ABILITY to vary over the five levels of:

$$
\begin{aligned}
& 1=\text { very low } \\
& 2=\text { low } \\
& 3 \text { = average } \\
& 4=\text { high } \\
& 5=\text { very high }
\end{aligned}
$$

While effort can take on the levels of:

$$
\begin{aligned}
& 1=\text { none } \\
& 2=\text { moderate } \\
& 3=\text { high }
\end{aligned}
$$

Our model, called GRADE1, can be expressed as:

\section{GRADE $=w_{1}$ * ABILITY effect $+w_{2} *$ EFFORT effect $+w_{3} *$}

ABILITY by EFFORT interaction + Constant + Error.

with the constraint that $0 \leqslant \mathrm{GRADE} \leqslant 100$.

To calculate the specific value, we must supply exact values for each term. Some values drawn from imagination (not based on published research) will be supplied to complete the example, although these values would normally be derived after either lots of research of the literature, or a rather complete knowledge built up with experience.

Since we assume that the effect of ABILITY alone or
EFFORT alone is negligible, then weights $w_{1}$ and $w_{2}$ are assumed equal to zero. Weight $\mathrm{w}_{3}$ is assumed to be 5 and the interactions are assumed to be:

\begin{tabular}{crrrrrr} 
& \multicolumn{7}{c}{ ABILITY } \\
& & 1 & 2 & 3 & 4 & 5 \\
& 1 & 0 & 0 & 0 & 1 & 2 \\
EFFORT & 2 & 0 & 2 & 4 & 6 & 6 \\
& 3 & 0 & 4 & 8 & 12 & 16
\end{tabular}

The constant is assumed to be 10 and the range of error is $20 \%$.

The entry of this model into the system in GMB mode is shown below. It should be emphasized that this model is only an example to give an idea of how the system works. For the sake of simplicity, the model shown here lacks the use of continuous variables, $X$ variables, secondary variables, and a cost function. In the author's guide, each of these is explained and added to GRADE1, each time adding a new model to the system which is more sophisticated.

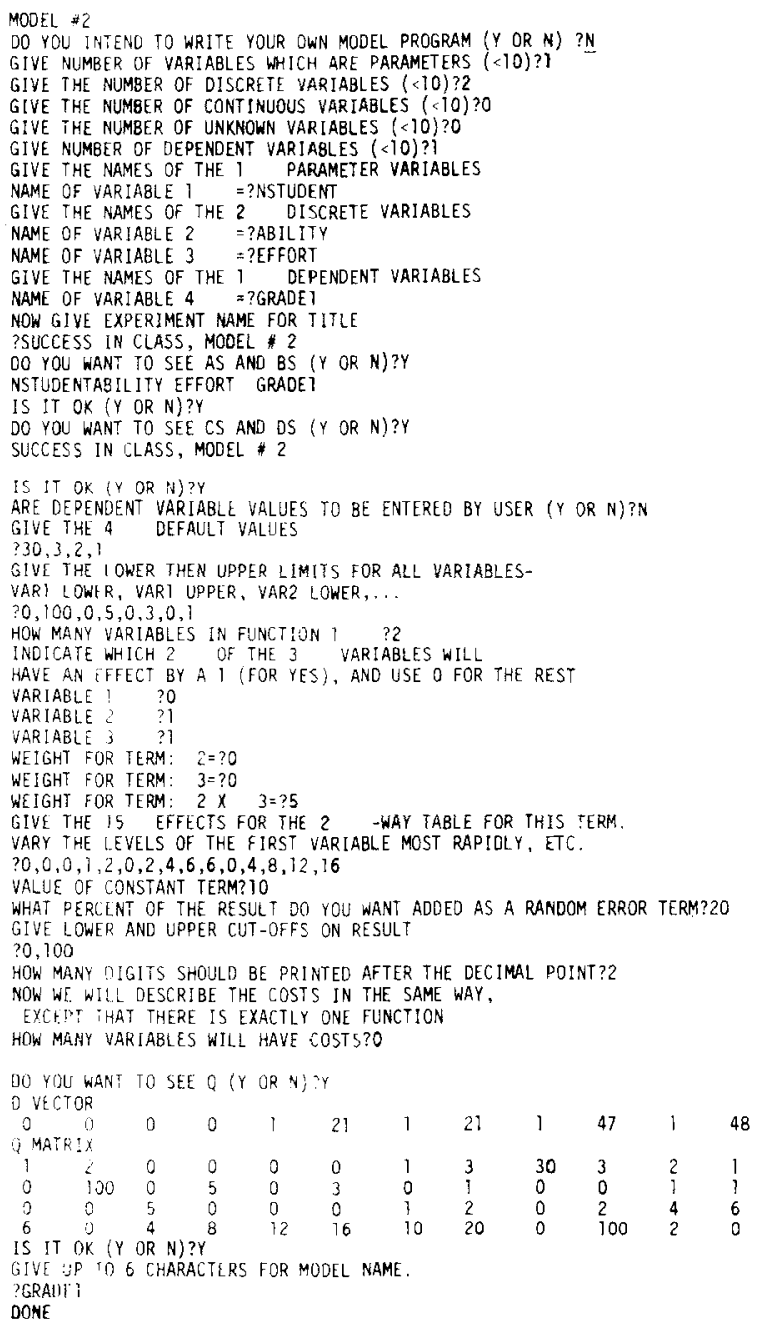


The running of this model is shown below. Note that the values are given horizontally, in a packed format, and there may be a perceptible pause before each value is printed, while it is being calculated.

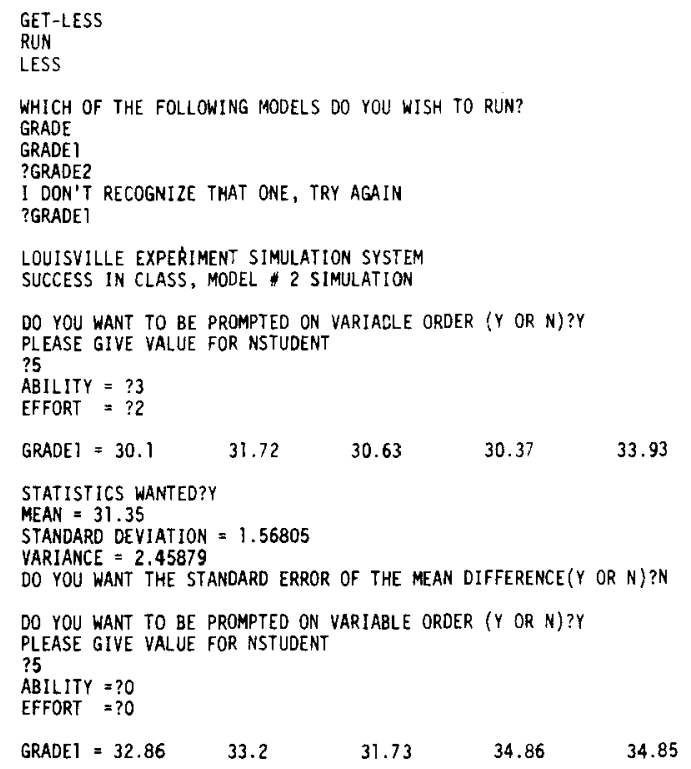

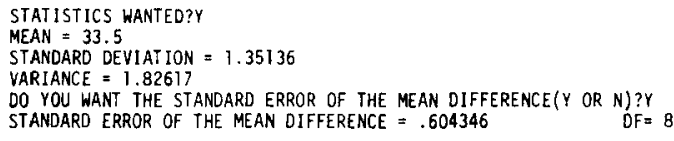

Note that when ABILITY $=0$ and EFFORT $=0$, the default values are assumed to be ABILITY $=3$ and EFFORT $=2$ since these were given when GMB was used alone to build the model. Thus, the data obtained on the second run differ from those obtained in the first run only because of random variation.

\section{REFERENCES}

Main, D. B., \& Head, S. Computer simulations in the elementary psychological laboratory. Proceedings of the Conference on Computers in the Undergraduate Curricula, Dartmouth, June 1971 .

Shure, G., \& Brainerd, K. MODELR: Model building and model modification for instruction. Behavior Research Methods \& Instrumentation. 1975. 7, 221-225.

Stout, $R$. Modeling on the simulation writer interactive program. Behavior Research Methods \& Instrumentation, 1975, 7, 226-228.

Thurmond, J. B., \& Cromer, A. O. Toward the optimal use of computer simulations in teaching scientific research strategy. Proceedings of the Conference on Computers in the Undergraduate Curricula, Atlanta, June 1972. 